PHYSICAL REVIEW B 95, 039904(E) (2017)

\title{
Erratum: Quantum heat engines based on electronic Mach-Zehnder interferometers [Phys. Rev. B 91, 195406 (2015)]
}

Patrick P. Hofer and Björn Sothmann

(Received 18 January 2017; published 30 January 2017)

DOI: 10.1103/PhysRevB.95.039904

There is an error in the current estimate obtained for a temperature bias of $60 \mathrm{mK}$ in Sec. IV. At the voltage that maximizes the power, the correct estimate is $I^{e} \approx 0.044 \mathrm{nA}$ for the three-terminal setup and $I^{e} \approx 0.053 \mathrm{nA}$ for the double Mach-Zehnder interferometer. We emphasize that this Erratum does not affect any other results and conclusions of our paper. 